\title{
A military garrison or cultural mixing pot? Renewed investigations at Shichengzi, a Han Dynasty settlement in Xinjiang
}

\author{
Pengfei Sheng ${ }^{1,2}$ (D), Michael J. Storozum ${ }^{1,2,3, *}$ (D) Xiaohong $\operatorname{Tian}^{4} \&$ Yong Wu ${ }^{4}$ \\ ${ }^{1}$ Institute of Archaeological Science, Fudan University, P.R. China \\ ${ }^{2}$ Department of Cultural Heritage and Museology, Fudan University, P.R. China \\ ${ }^{3}$ Department of Archaeology, Max Planck Institute for the Science of Human History, Germany \\ ${ }^{4}$ Xinjiang Institute of Cultural Relics and Archaeology, Urumqi, P.R. China \\ * Author for correspondence $\bowtie$ mjstorozum@fudan.edu
}

\begin{abstract}
Excavations at Shichengzi, the probable location of ancient Shule, have revealed diverse burial practices suggesting a population with varied cultural backgrounds. Together with archaeobotanical evidence, this indicates a community of agro-pastoralists and Han Dynasty migrants using diverse cropping patterns to attain selfsufficiency. The project raises interesting questions about the impact of migrations on the identities of inhabitants.
\end{abstract}

Keywords: China, Han Dynasty, tuntian, migrations, farming

New research on the ancient 'Silk Road' is revealing how trade and exchange with peoples living in Central Asia played a pivotal role in supporting both the Roman (27 BC-AD 476) and Han (202 BC-AD 8 \& AD 25-220) Empires (Scheidel 2009; Spengler 2019). While the Roman Empire derived revenue from indirect trade with Central Asian kingdoms, the Han Dynasty sought to influence the steppe directly by projecting a combination of military and economic power (Scheidel 2015). Specifically, in 101 BC Emperor Han Wu Di (汉武帝) ordered thousands of people to migrate from the North China Plain to establish tuntian (屯田) outposts. These were large, self-sufficient military garrisons at the western fringes of the Han Dynasty's empire, which served as political, military and economic bulwarks against the agro-pastoral kingdoms of Central Asia (Zhang \& Tian 2015).

Although both the Han Shu (The History of the Former-Han) and the Hou Han Shu (The History of the Latter-Han) briefly reference tuntian settlements (Ban 1962; Fan 2000), specific details about the economy and innerworkings of these frontier towns can only be revealed by archaeological investigations of these ancient settlements. In 2007, the Xinjiang Institute of Cultural Relics and Archaeology began a formal survey to catalogue all known archaeological sites in the province as a starting point for future research programmes (Bureau of Cultural Relics of Xinjiang Uyghur Autonomous Region 2011). Thus far, they

Received: 24 June 2019; Revised: 19 September 2019; Accepted: 8 October 2019

(C) The Author(s), 2020. Published by Cambridge University Press on behalf of Antiquity Publications Ltd. This is an Open Access article, distributed under the terms of the Creative Commons Attribution licence (http://creativecommons. org/licenses/by/4.0/), which permits unrestricted re-use, distribution, and reproduction in any medium, provided the original work is properly cited. 


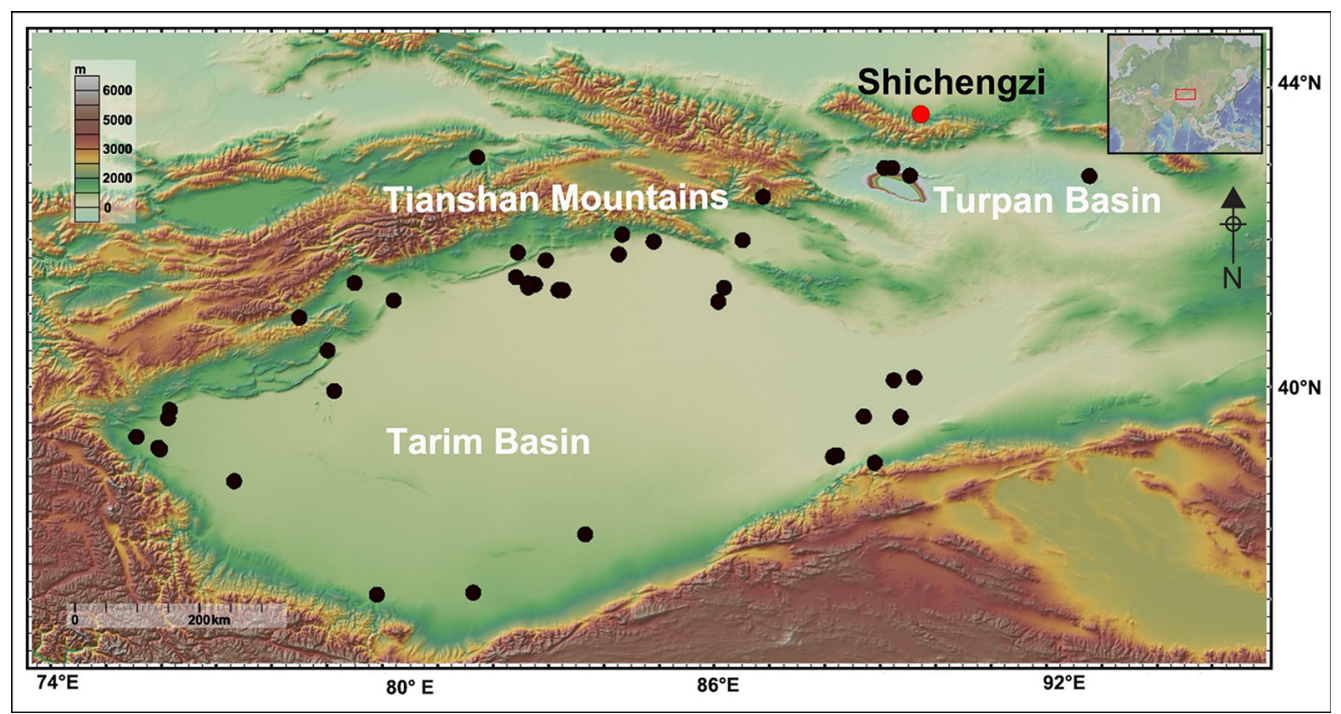

Figure 1. Map of Xinjiang Han city sites including Shichengzi (red dot). Map by Pengfei Sheng.

have found at least 45 sites dating to the Han Dynasty that each have the potential to reveal new insights into the colonisation of its western frontier (Figure 1).

In recent years, archaeologists have re-opened excavations at one of these sites, Shichengzi, which is the probable location of Shule (疏勒城); Shule was a Han Dynasty garrison, which, according to the Hou Han Shu, was abandoned in AD 75 several decades after the collapse of the western Han Dynasty (Dai 1995; Fan 2000). Assuming the chronology presented in the Hou Han Shu is correct, Shule's short-lived occupation enables archaeologists to examine the effects of the forcible migration of Han Dynasty people into Central Asia and the impact of these migrations on the local economy, the identity of the native peoples of the region and the migrants themselves.

Members of a new collaborative project between the Institute of Archaeological Science at Fudan University and the Xinjiang Provincial Institute of Cultural Relics and Archaeology are currently using archaeobotanical, zooarchaeological and isotopic analyses on materials recovered from Shichengzi to address two questions that guide this project:

1. How did the Han migrants at Shichengzi produce enough food to sustain the garrison?

2. What were the interactions between the Han migrants and agropastoralist peoples in the area?

\section{The Shichengzi site}

Shichengzi $\left(89^{\circ} 45^{\prime} 43.2^{\prime \prime} \mathrm{E}, 43^{\circ} 36^{\prime} 59.1^{\prime \prime} \mathrm{N} \& 1770 \mathrm{~m}\right.$ asl) was first discovered in 1973 and further explored during the second and third National Survey of Cultural Relics in Xinjiang in 1988 and 2009 (Figure 1). During these surveys, archaeologists found rammed earth walls, pottery, tiles, bricks and other artefacts that had accumulated on a hillside at Shichengzi, 

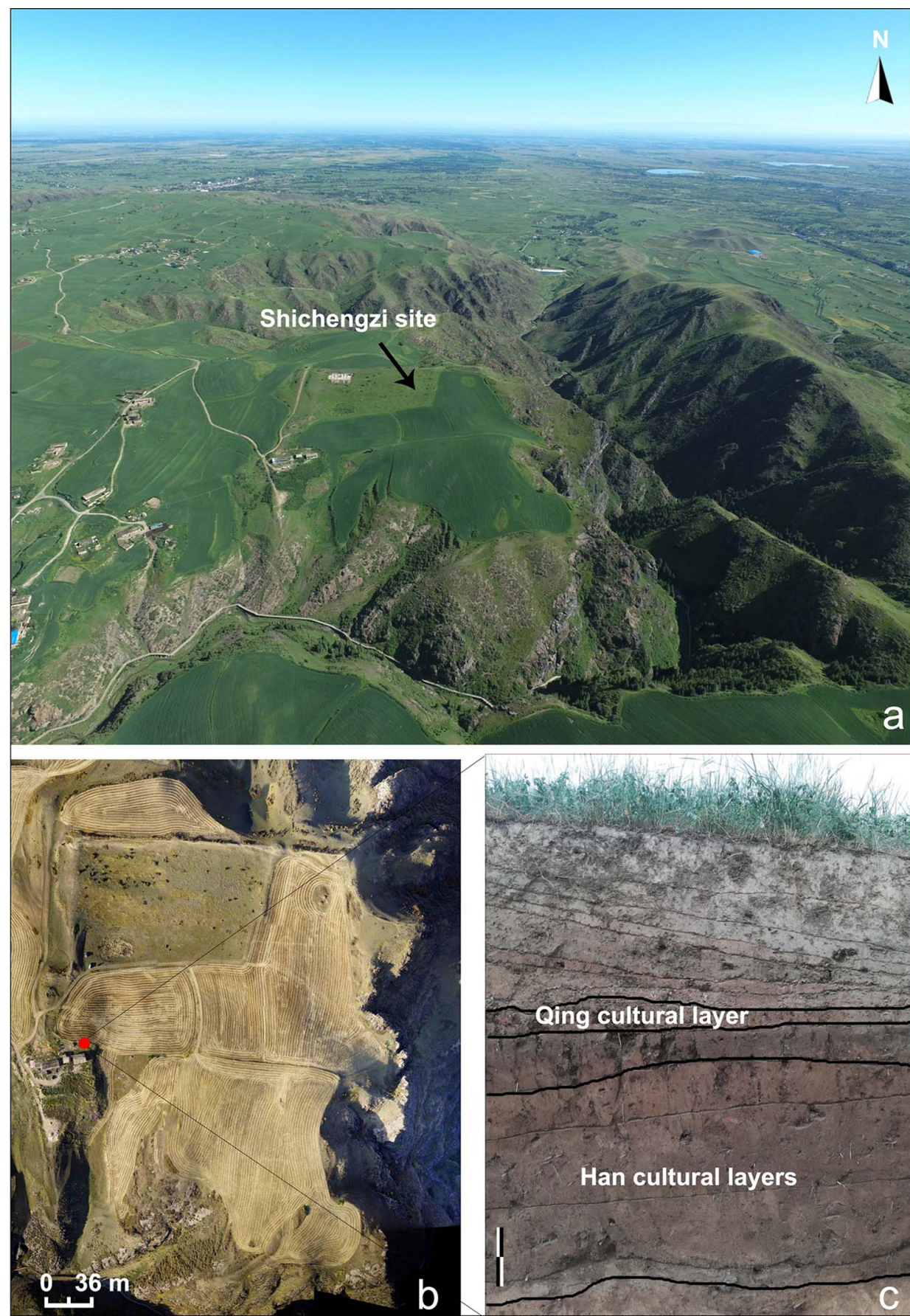

Figure 2. a) A panoramic view of Shichengzi (black arrow) and the natural vegetation of the surrounding area; $b$ ) aerial photograph of the area around Shichengzi; c) a stratigraphic profile at Shichengzi. Scale bar $=0.2 \mathrm{~m}$. Figure provided by Xiaohong Tian. 

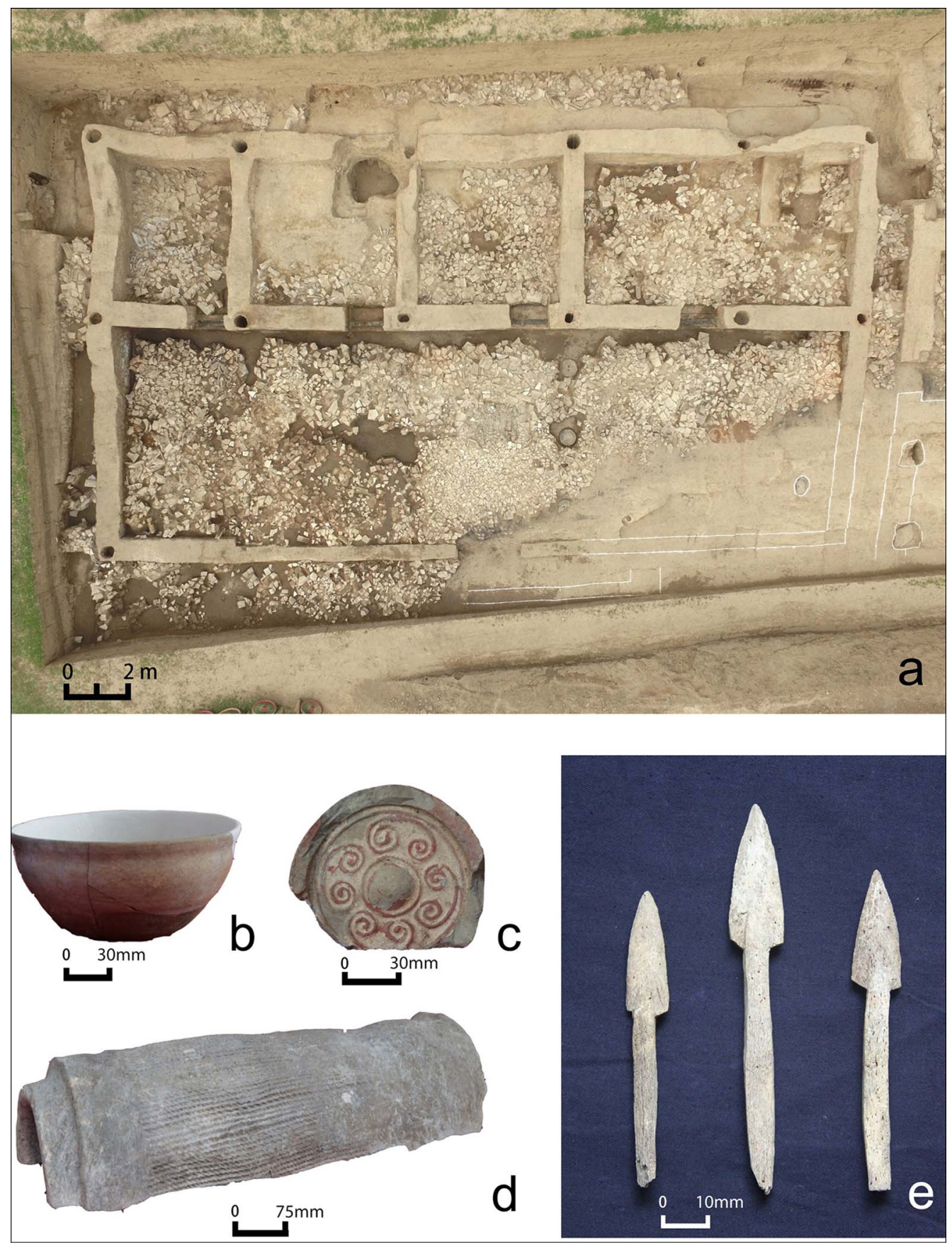

Figure 3. a) A series of rooms excavated at Shichengzi; b) a pottery bowl (ID: F1c: 4); c) the Wadang (clay roof tile end) with typical Han style (ID: F1a: 5); d) the pottery tile (ID: F1b: 10), decorated with rope and fabric textures on the surface; e) bone arrows (ID: F1x: 7). Figure provided by Xiaohong Tian.

(C) Antiquity Publications Ltd, 2020 


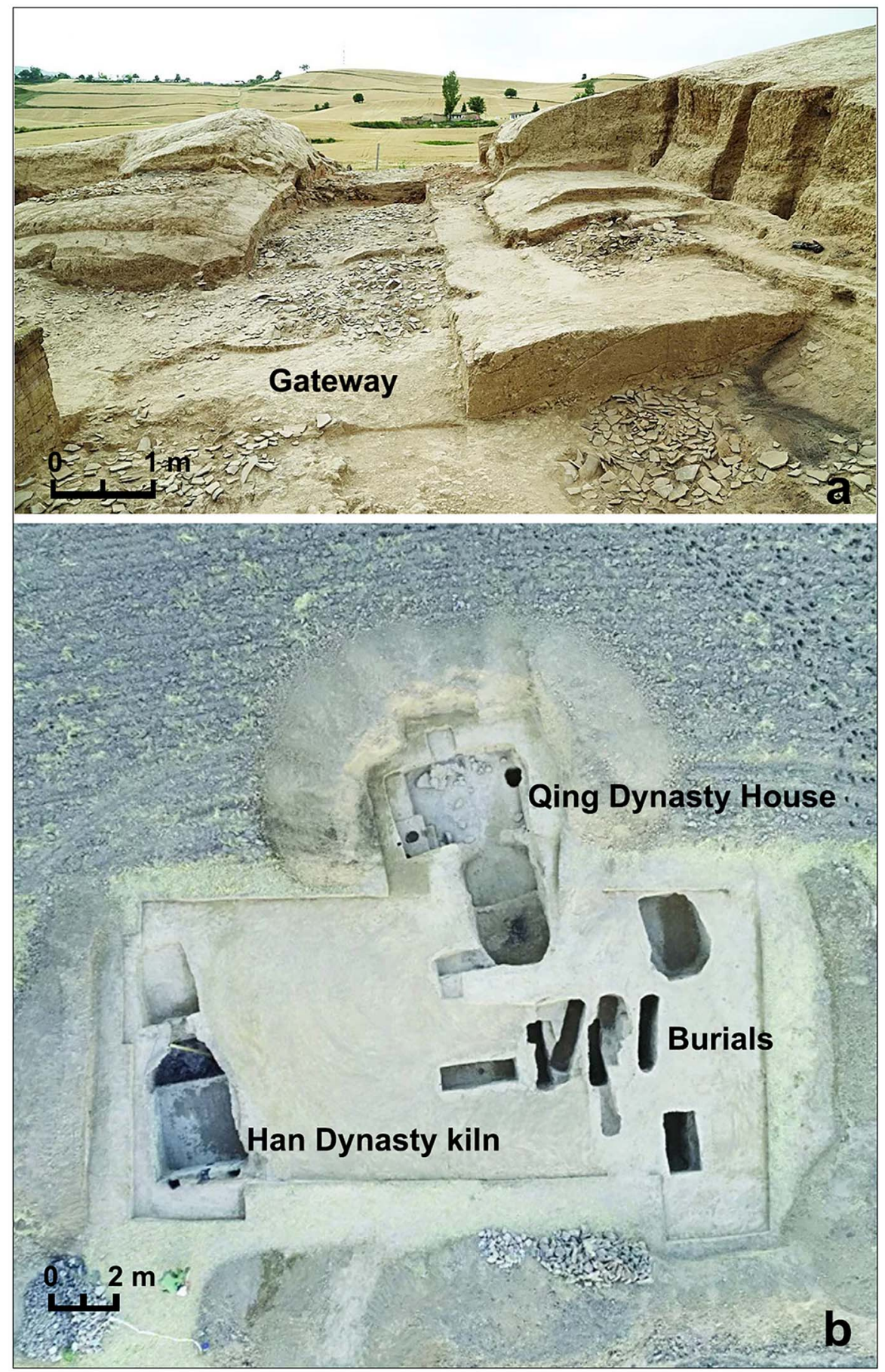

Figure 4. a) The gate and gateway of Shichengzi; b) burials and kiln found at Shichengzi. Photograph by Xiaohong Tian \& Yong Wu. 


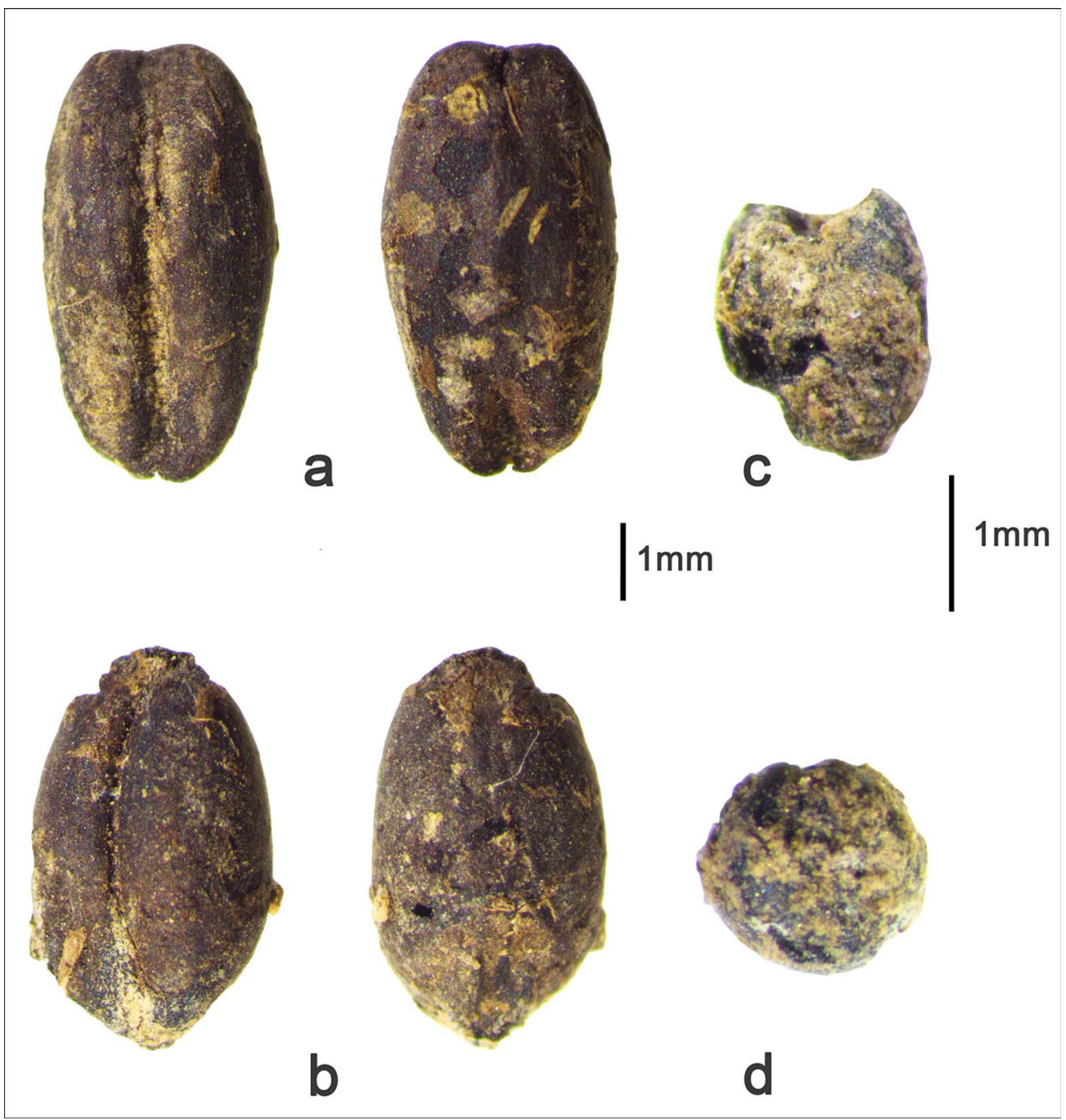

Figure 6. Charred crops discovered at Shichengzi: a) naked barley (Hordeum vulgare var. coeleste); b) bread wheat (Triticum aestivum); c) common millet (Panicum miliaceum); d) foxtail millet (Setaria italica). Photograph by Pengfei Sheng.

indicating that the site had abundant material culture. Scientific excavations did not start until 2014, however (Figure 2a \& b). Since the start of these renewed excavations, archaeologists have found that the site is comprised of two main phases dating to the Han (202 BC$\mathrm{AD} 8 \& \mathrm{AD} 25-220$ ) and Qing Dynasties (AD 1636-1911) (Figure 2c). The city area is $110000 \mathrm{~m}^{2}$, about $380 \mathrm{~m}$ long and $280 \mathrm{~m}$ wide, surrounded by the remnants of earthen walls (Figure 2b). Archaeologists have also found substantial architectural remains, including the main gate of the city, moats, houses, storage pits and even a kiln (Figures 3-4). In the coming years, the Xinjiang Institute of Cultural Relics and Archaeology will continue their excavations to reveal the site's plan (Figure 5). 
In 2018, ten burials believed to belong to a culturally diverse group of people were discovered in the western portion of the site, potentially providing new insights into who the inhabitants of the site at Shichengzi were (Figure $4 \mathrm{~b}$ ). Six of the burials were pit tombs, three were pit tombs with partial chambers at the bottom, and one was a pit tomb with a platform at the base. The tomb with the platform contained many calcaneal sheep (Ovis aries) bones and half of the skeleton of a horse (Equus caballus). The individuals in the tombs were all buried in the prone position in wooden coffins, facing west. Many grave goods were discovered within these tombs, such as bronze rings, earrings, bracelets and two wuzhu (五铢) coins diagnostic of the western Han Dynasty, which hint that individuals buried at the site at Shichengzi were a mix of agro-pastoralists and Han migrants.

Preliminary results from the flotation of deposits collected from several mud-brick houses within the city walls and from storage pits have revealed four types of charred cereal grains, including naked barley (Hordeum vulgare var. celeste), bread wheat (Triticum aestivum), common millet (Panicum miliaceum) and foxtail millet (Setaria italica) (Figure 6). The mixed assemblage suggests that inhabitants of the site at Shichengzi practised a diverse cropping pattern oriented towards risk mitigation. Our initial results indicate that naked barley grains are the most abundant and ubiquitous type of seed found at the site, suggesting that the cultivation of naked barley was the core agricultural strategy. Naked barley is very adaptable to high elevations and cold environments, making it an ideal crop for cultivation around Shichengzi. These results reveal that the people who lived at and around the site at Shichengzi adopted an agricultural strategy adapted to the environment around the northern slopes of the Tianshan Mountains.

\section{Conclusion}

The collaboration between Xinjiang's Institute of Cultural Relics and Archaeology and Fudan University is beginning to provide answers to many previously unknown aspects of these short-lived Han Dynasty settlements. Our preliminary results have already revealed that the garrison colony at Shichengzi probably had a mix of agro-pastoralist peoples and Han migrants. It is likely that these people practised a risk-averse agricultural strategy that was heavily reliant on naked barley. Many questions remain unanswered and further archaeological research at Shichengzi is necessary to reveal the farming methods used by Han Dynasty migrants in their new surroundings. It is hoped that future research will also shed light on how Han migrants interacted with the local peoples and whether they retained their connection to the distant imperial cities in central China.

\section{Acknowledgements}

This study was funded by a China Postdoctoral Science Foundation Grant (2018M641902 \& 2019M651399).

\section{References}

Ban, G. 1962. Han Shu. Beijing: China Publishing House (in Chinese).
Bureau of Cultural Relics of Xinjiang Uyghur Autonomous Region (ed). 2011. Ancient city 
relics in Xinjiang. Beijing: Cultural Relics (in Chinese).

DAI, L. 1995. Debate of the location of Shule: current evidence from the Shichengzi site in Qitai of Xinjiang. China's Borderland History \& Geography Studies 4: 93-95 (in Chinese).

Fan, Y. 2000. Hou Han Shu. Beijing: China Publishing House (in Chinese).

SCHeIdel, W. 2009. Rome and China: comparative perspectives on ancient world empires. Oxford: Oxford University Press.
Scheidel, W. (ed.). 2015. State power in ancient China and Rome. Oxford: Oxford University Press.

SPEngler III, R.N. 2019. Fruit from the sands: the Silk Road origins of the foods we eat. Berkeley: University of California Press. https://doi.org/10.2307/j.ctvh1dx4s

Zhang, A. \& H. Tian. 2015. The remains of the site layout of the defence cities of the Han Dynasty in the western regions. Journal of Chinese Historical Geography 30(3): 47-55 (in Chinese). 